\title{
The Optimal Hydraulic Design of Centrifugal Impeller Using Genetic Algorithm with BVF
}

\author{
Xin Zhou, Yongxue Zhang, Zhongli Ji, and Hucan Hou \\ College of Mechanical and Transportation Engineering, China University of Petroleum, Beijing 102249, China \\ Correspondence should be addressed to Xin Zhou; ryanzhou@yeah.net
}

Received 25 January 2014; Revised 2 April 2014; Accepted 28 May 2014; Published 20 August 2014

Academic Editor: Ryoichi Samuel Amano

Copyright ( 2014 Xin Zhou et al. This is an open access article distributed under the Creative Commons Attribution License, which permits unrestricted use, distribution, and reproduction in any medium, provided the original work is properly cited.

\begin{abstract}
Derived from idea of combining the advantages of two-dimensional hydraulic design theory, genetic algorithm, and boundary vorticity flux diagnosis, an optimal hydraulic design method of centrifugal pump impeller was developed. Given design parameters, the desired optimal centrifugal impeller can be obtained after several iterations by this method. Another 5 impellers with the same parameters were also designed by using single arc, double arcs, triple arcs, logarithmic spiral, and linear-variable angle spiral as blade profiles to make comparisons. Using Reynolds averaged N-S equations with a RNG $k$ - $\varepsilon$ two-equation turbulence model and log-law wall function to solve 3D turbulent flow field in the flow channel between blades of 6 designed impellers by CFD code FLUENT, the investigation on velocity distributions, pressure distributions, boundary vorticity flux distributions on blade surfaces, and hydraulic performance of impellers was presented and the comparisons of impellers by different design methods were demonstrated. The results showed that the hydraulic performance of impeller designed by this method is much better than the other 5 impellers under design operation condition with almost the same head, higher efficiency, and lower rotating torque, which implied less hydraulic loss and energy consumption.
\end{abstract}

\section{Introduction}

Centrifugal pump has extensive applications in industry and other technical sectors, because of its design simplicity, high efficiency, smooth flow rate, and ease of operation and maintenance. Among all the installed pumps in a typical petroleum plant, almost $80-90 \%$ pumps are centrifugal type. A wealth of experience of centrifugal pump impeller hydraulic design is accumulated and the efficiency of centrifugal pump has reached a considerable level $[1,2]$. But due to the great number of free geometric parameters involved, its design and optimal processes are still difficult tasks. The design heavily relies on the experience of the engineer to select and evaluate the many possible geometrical variations. These manual processes are often time consuming and multiple "trial and error" procedures are needed to identify the right combination for the best solution. Often a thorough exploration of the solution space to find the optimal design for the real life problem is hardly possible. In recent years, many modern pump design methods have been developed and with the help of some software such as TURBOdesign, CFTurbo, and Pcad, pump design and optimal processes become simpler. But these is still some work needed to be done to find the connection between the hydraulic performance of pump and its inner local flow field, which will guide to improve its performance more accurately.

Genetic algorithm (GA) is a global search technique that mimics Darwin's theory of biological evolution used in computing to find exact or approximate solutions to optimization and search problems, which is inspired by evolutionary biology such as inheritance, selection, crossover, and mutation. Unlike classical methods, it is not affected by local optima. Genetic algorithm is first presented by John Holland in the early 1970s and it is currently the most popular design optimization algorithm. GA can be used to find out the optimization even if the objective function does not have a derivative or if it is very hard to calculate its derivative. Thus, it is mostly used for design parameter optimization, shape optimization, or topology optimization [3-5]. But when it comes to complex problems, repeated fitness function evaluation will be the most prohibitive and limiting part of GA. The counterweight to the increased computing requirements will be used as an efficient evaluation model. 
With the purpose of using efficient and simple inviscid flow model, some assistance from boundary vorticity dynamics is introduced and boundary vorticity flux (BVF) is used to diagnose the flow in impeller channels. BVF measures the vorticity creation rate from solid surface and enables a significant focus on localized key regions, and from theory it shows that the total force and moment can be cast to proper surface integrals of BVF no matter how complicated the vorticity evolution is, which can be managed and controlled by proper design [6-8]. Since the mid-1980s, Wu et al. have established a theory of boundary vorticity dynamics and successfully applied this theory to the aerodynamic diagnosis and optimization of airfoil profile [9]. Zhang et al. optimized the impeller by adjusting the geometrical parameters and modifying the meridional shape of the blade based on BVF method, and the hydraulic performance of the pump was improved [10]. Wu et al. fully used the advantage of BVF to detect the area during blade design and modified the blade profile under the guidance of flow theory of impeller [11]. Zhang et al. have reviewed vortex dynamics theory with emphasis on its physical background and demonstrated its applications [12].

Regardless of the design process used, the final decision of new pump impeller design is usually made following physical prototypes testing. Conducting these processes in which extremely advanced measuring techniques or even visualization techniques should be employed is often time and resources consuming. In addition, no simple mathematic model can be established to well predict the performance of impellers at present and by using numerical procedures it is possible to predict the performance curve of impellers with enough accuracy. For these reasons computational fluid dynamics analysis is currently being used in hydrodynamic design for centrifugal impellers [13-15]. The development of CFD that compute the flow field inside the impeller has been a breakthrough in the field of design, but it do not automatically realize an optimal design by itself.

In this paper, an optimal hydraulic design method of centrifugal pump impeller which combines the advantages of two-dimensional hydraulic design theory, genetic algorithm, and boundary vorticity flux diagnosis is presented. Another 5 impellers with the same design parameters have also been designed as comparison and the three-dimensional turbulent flow fields of these impellers are calculated numerically by commercial CFD code. Comparisons of flow fields in these impellers and prediction of their hydraulic performance are also presented.

\section{GA Hydraulic Design Method}

Since a great number of free geometric parameters involved in the impeller hydraulic design and multiple "trial and error" procedures which will be conducted with much experience are inevitable, the hydraulic designs of centrifugal impeller are always tedious and time and resource consuming. With the purpose of simplifying design, reducing the time taken by design, and making use of the merit of global search technique from GA, a hydraulic design method of impeller based on two-dimensional flow theory design and GA is presented.

The flow procedure of GA hydraulic design is shown in Figure 1. Firstly, a population of chromosomes of given size $N$ has been generated; the values of design parameters and control parameters will be obtained by decoded chromosomes. After that the hydraulic design process is presented to create a new impeller, the estimated BVF distribution on designed blade surfaces can be calculated during the design procedure, and its values can be used in an evaluation function which will help to gain the fitness of each chromosome. And then the GA iteration procedures including selection, crossover, mutation, and replacement are carried out until the stop criterion is fulfilled.

2.1. Two-Dimensional Theory Hydraulic Design Method. The hydraulic design method is based on some hypothesis of twodimensional flow theory, the working medium is considered as inviscid and incompressible fluid, and the flow is axial symmetric about rotating axis with consideration that infinite blades are setting on the impeller channel. According to the desired operating conditions (usually capacity, head, rotating speed, etc.), the basic geometrical features of the initial impeller (such as $D_{1}, D_{2}, B_{2}$, and $Z n$ ) can be determined by empirical correlations which are based on the method of velocity coefficient. And after that the meridional section profile of impeller can be generated with curves. Four points Bezier curves are used here to draw both the hub and shroud profile lines, as shown in Figure 2. The starting and ending points of these curves can be fixed after the basic geometrical features are obtained, and changing the profile of meridional plane of impeller will be realized by moving each of two control points on hub and shroud curves.

When the meridional profile is ready, quasiorthogonal method is adopted on the meridional section to calculate the flow field in impeller. The established quasiorthogonal gradient equation of meridional component of absolute velocity can be solved by point-by-point integration method. More specific details of these procedures can be found in paper $[16,17]$.

The equation is as follows:

$$
\begin{aligned}
\frac{d C_{m}}{d s}= & C_{m}\left[\left(\frac{d \alpha_{1}}{d s}-\frac{\partial \alpha_{1}}{\partial l} \sin \delta\right) \frac{1}{\cos \delta}-\frac{\sin \alpha_{1}}{r}-\frac{\partial \ln \psi}{\partial l}\right] \\
& \times \sin \delta+K \cos \delta
\end{aligned}
$$

where $K=-k C_{m}\left(d \alpha_{1} / d l\right)$, and its value of parameter $K$ should be given to solve the equation to get meridional velocity $C_{m}$. This equation represents one-dimensional flow theory design at the condition $k=0$, two-dimensional flow theory design with $\Omega_{u}=0$ at the condition $k=1$, and twodimensional flow theory design with $\Omega_{u} \neq 0$ at the condition $k \in(0,1)$. The relation between quasiorthogonal, streamline, and cross-section line is shown in Figure 3.

Assuming that impeller has infinite number of blades, the camber lines of blades and streamlines are coincided. Given the relationship between blade wrapping angle and length 


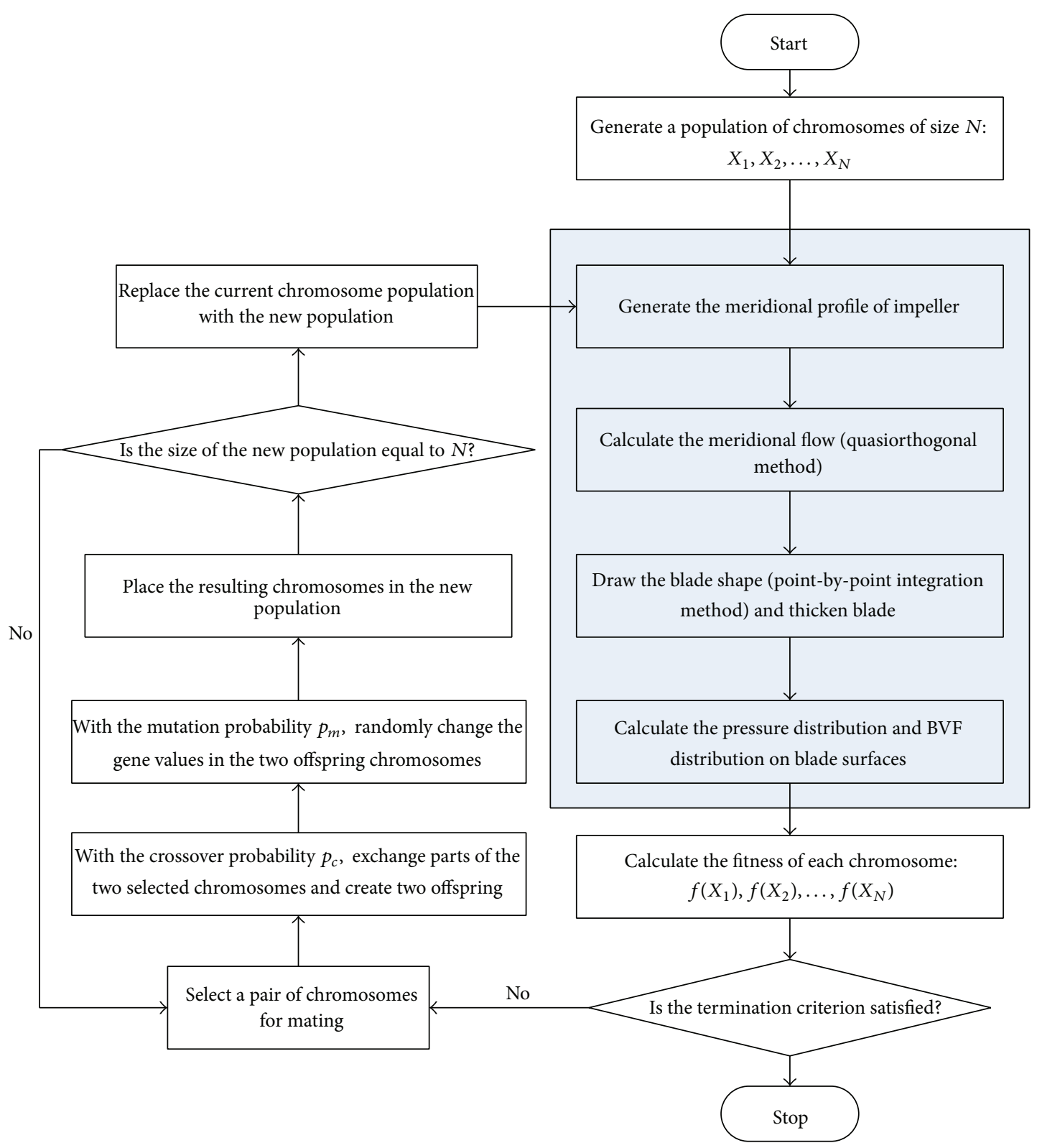

FIGURE 1: Flowchart of GA hydraulic design.

of meridional streamline, camber lines can be obtained by solving the integral of wrapping angle function according to a given relative velocity distribution along the streamline:

$$
\varphi=\int_{0}^{l} \frac{1}{r} \sqrt{\frac{W^{2}}{C_{m}^{2}}}-1 d l .
$$

Since the leading edges are concerned with cavitation and instabilities of head-capacity characteristic curve of the pump, during the design process more attention should be paid to them. When the procedure of blade drawing has been finished, the value of function $\varphi_{i}=\varphi_{i}(l)$, where $l$ is the streamline length along the flow direction and the suffix means $i$ th streamline on the blade, at which point on the blade profile can be gained (define $\varphi_{0 i}$ as the wrapping angle calculated at each streamline on the blade). Assuming that the positions of trailing edges of blades remain fixed (certainly, the positions of trailing edges can be given as demand at first), if the required wrapping angle $\varphi_{0 i}^{\prime}$ on each streamline is given, the required leading edges will be $\varphi_{l i}^{\prime}=\varphi_{t i}-\varphi_{0 i}^{\prime}$ and the position of them can be easily determined by setting up equation of the relationship between $\Delta \varphi$ and $\Delta l$. Iterations are needed to insure that the wrapping angles on each streamline after leading edges changing are really equal to the required ones.

After leading edges change, the meridional flow should be recalculated. Through this method, the free geometric parameters of leading edges of blades can be reduced and connection between the position of leading edge and trailing edge by wrapping angle is obtained, which brings a more 


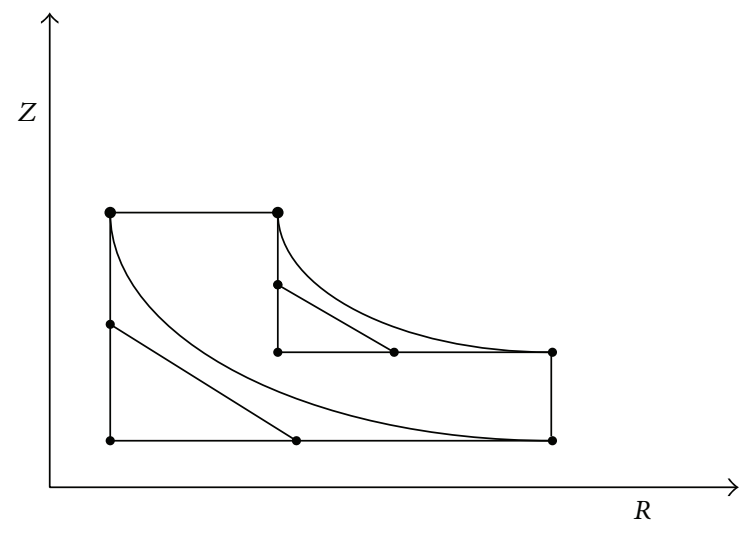

FIGURE 2: Meridional profile of impeller.

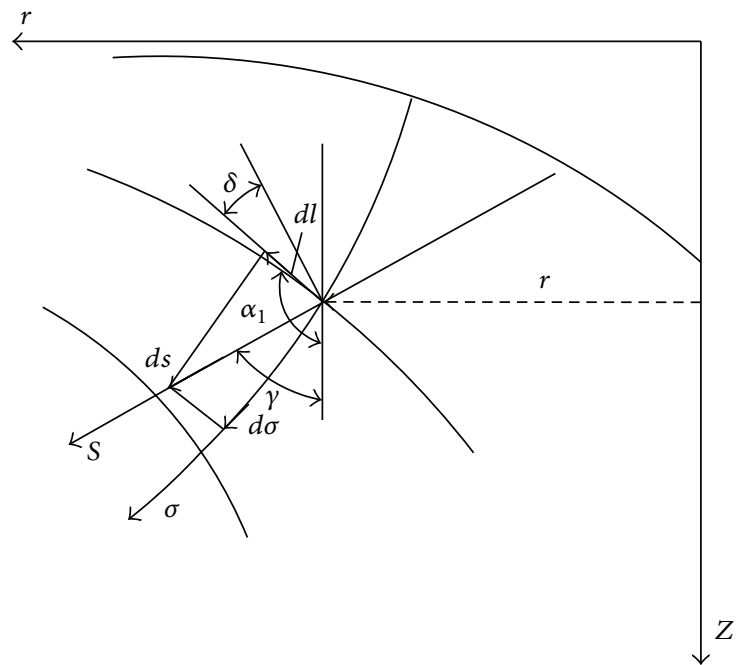

FIGURE 3: The relation between quasiorthogonal, streamline, and cross-section line.

convenient way to design the leading edge. In this hydraulic design procedure, after obtaining the basic geometrical features of the initial impeller (such as $D_{1}, D_{2}, B_{2}$, and $Z n$ ), only 4 control points (which will be given to generate the meridional profile), incidence angles at leading edges and deviation angle at trailing edges of blades, meridional flow factor $k$, relative velocity along the meridional streamline, wrapping angles on each streamline, and thickness distribution along the blade camber line should be known to realize an impeller design.

2.2. Boundary Vorticity Flux Diagnosis. The values of BVF measure the vorticity creation rate from solid wall surface, and the surface part will have a net contribution to the total force and moment if it creates vorticity [6], so that, through BVF diagnosis, a few localized key regions of blade surfaces will be much more easily identified than normal method through pressure analysis.
For a Newtonian fluid as water, an intrinsic triple decomposition of the stress tensor will be expressed as [7]

$$
\mathbf{T}=-\Pi \mathbf{I}+2 \mu \Omega-2 \mu \mathbf{B},
$$

where $\lambda, \mu$ are the first and second viscosities, $\Pi=p-(\lambda+$ $2 \mu) \vartheta$, dilatation $\vartheta=\nabla \cdot \mathbf{u}$, the antisymmetric spin tensor $\Omega_{i j}=0.5 \varepsilon_{i j k} \omega_{k}$, and surface-strain rate tensor $\mathbf{B}=9 \mathbf{I}-(\nabla \mathbf{u})^{T}$ (with $\nabla \cdot \mathbf{B}=0$ ). And for a constant $\mu$, the divergenceless $\mathbf{B}$ disappears from the motion equation

$$
\nabla \cdot \mathbf{T}=-\nabla \Pi-\mu \nabla \times \boldsymbol{\omega} .
$$

The total force acting on a closed boundary is

$$
\mathbf{F}=-\int_{\partial D} \mathbf{n} \cdot \mathbf{T} d S=-\int_{\partial D}(\Pi \mathbf{n}+\mathbf{n} \times(\mu \boldsymbol{\omega})) d S .
$$

In three-dimensional space, based on the generalized Stokes theorem, the above expression can also be written as

$$
\mathbf{F}=-\frac{1}{2} \int_{\partial D} \mathbf{r} \times(\mathbf{n} \times \nabla \Pi) d S-\int_{\partial D} \mathbf{r} \times[(\mathbf{n} \times \nabla) \times(\mu \boldsymbol{\omega})] d S .
$$

As it can be seen from the total force expression, what really matters is only the tangent variation of $\Pi$ and $\mu \omega$ over surface $\partial D$. And if the following notation is used,

$$
\begin{gathered}
\boldsymbol{\sigma}_{\Pi} \equiv \frac{1}{\rho} \mathbf{n} \times \nabla \Pi, \\
\boldsymbol{\sigma}_{\omega} \equiv \frac{1}{\rho}(\mathbf{n} \times \nabla) \times(\mu \boldsymbol{\omega})
\end{gathered}
$$

the expression will change to

$$
\mathbf{F}=-\frac{1}{2} \int_{\partial D} \rho \mathbf{r} \times \boldsymbol{\sigma}_{\Pi} d S-\int_{\partial D} \rho \mathbf{r} \times \boldsymbol{\sigma}_{\omega} d S .
$$

At large Reynolds number conditions, the effect of explicit viscous terms is a few orders smaller than that of tangent pressure gradient. For BVF diagnosis, it is sufficient to focus only on

$$
\boldsymbol{\sigma}_{P}=\frac{1}{\rho} \mathbf{n} \times \nabla P .
$$

Any changes in the location and magnitude of BVF distribution can be accounted as redistribution of pressure on surfaces. Since $\boldsymbol{\sigma}_{P}$ is a direct consequence of no-slip wall condition, it is only in viscous flow that $\boldsymbol{\sigma}_{P}$ is available; however, when the Reynolds numbers are large enough (it usually happens in the flow of impeller passages), $\sigma_{P}$ can be well estimated from an inviscid flow solution. And that is why it can be used within inviscid two-dimensional hydraulic design method.

Using the hydraulic design procedure mentioned above, the inviscid flow in impeller flow passage will be obtained after its design. In order to calculate values of BVF, the pressure distribution should be known firstly. Applying Bernoulli equations along each streamline on the blade surfaces,

$$
\frac{W^{2}-U^{2}}{2}+g Z+\frac{P}{\rho}=\text { Const. }
$$




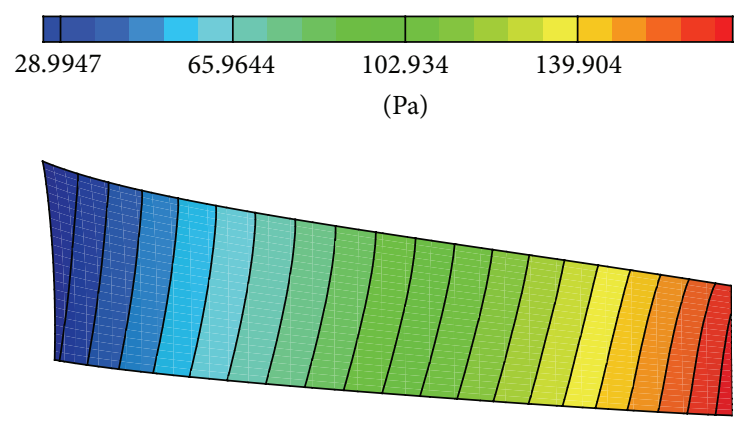

FIgURE 4: Pressure distribution.

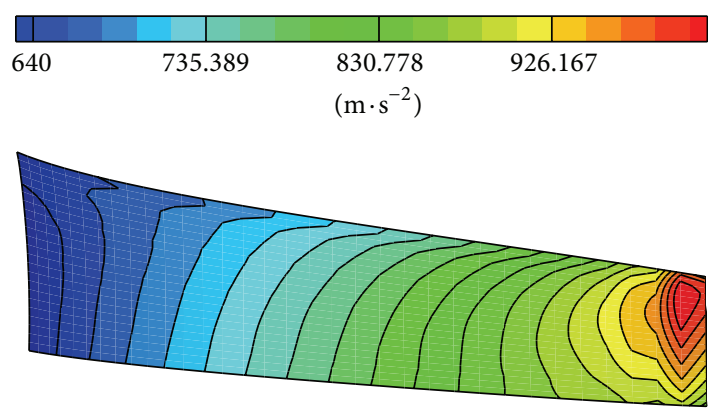

Figure 5: BVF distribution.

Once the reference pressure is given, the distributions of pressure on blades can be assured, as shown in Figure 4 (pressure distribution on blade surface of one designed impeller). For the centrifugal pump impeller, $\sigma_{P z}=\boldsymbol{\sigma}_{P} \cdot \mathbf{e}_{z}$ which is the $Z$ direction component of $\sigma_{p}$ plays an important role in contributing to moment. At each point on the blade surface, the tangent vector of streamline $\mathbf{l}$, the tangent vector of cross-section line $\mathbf{m}$, normal vector direction $\mathbf{n}$ from an orthogonal curve coordinate, and the value of $\sigma_{P z}$ can be calculated using the following expression:

$$
\sigma_{P z}=\boldsymbol{\sigma}_{P} \cdot \mathbf{e}_{z}=\left(\frac{1}{\rho} \mathbf{n} \times\left(\frac{\partial P}{\partial l} \mathbf{e}_{l}+\frac{\partial P}{\partial m} \mathbf{e}_{m}+\frac{\partial P}{\partial n} \mathbf{e}_{n}\right)\right) \cdot \mathbf{e}_{z},
$$

where $\partial P / \partial l$ and $\partial P / \partial m$ can be calculated by using spline function or some other methods after pressure distribution on surface is known, as shown in Figure 5 as an example.

Since inviscid flow calculation is enough to obtain BVF information that is needed to check the performance of the designed blade, time consuming 3D CFD simulations are not inevitable and localized key regions will be more easily recognized as shown in Figure 5 (it shows that BVF peaks exist around trailing edge of blade). Through this, the comparisons between different design parameters can be more efficiently and accurately conducted.

2.3. Genetic Algorithm. Genetic algorithm (GA) is a global search technique used in computing to find exact or approximate solutions to optimization and search problems, which is inspired by evolutionary biology such as inheritance, selection, crossover, and mutation. GA can also be used to find out the optimization even if the objective function does not have a derivative or if it is very hard to calculate its derivative. But when it comes to complex problems, repeated fitness function evaluation will be the most prohibitive and limiting part of GA.

GA procedures begin with a randomly initialized population of chromosomes. These chromosomes are representations of the problem to be solved. An evaluation function is used to calculate the "goodness" of each chromosome and a fitness function is defined over the representations and measures the quality of these solutions. The selection of chromosomes for survival and combination is according to the fitness values of chromosomes. The fittest ones will have the most changes to exist in the next generation or become the parents of the next generation. Mutation, crossover, and inversion operators will be used to generate new chromosomes in the next generation. Repetitive application of these operators will be done to improve the solutions until the maximum generation number is reached or the desired criterion is met.

As for the hydraulic design of centrifugal impeller, the parameters (4 Bezier control points positions, incidence angle at leading edges and deviation angle at trailing edges of blades, meridional flow factor $k$, relative velocity distribution along the meridional streamline factor, and wrapping angles on each streamline) used in design procedure are encoded to chromosome and a function of BVF is constructed to be used as evaluation function. With the consideration of reducing the BVF peak values on the blade surfaces, the mathematical model is used as follows.

Objective functions are as follows:

$$
F\left(x_{1}, x_{2}, \ldots, x_{n}\right)=\min \left(\sum_{i=1}^{n}\left|B V F_{z}^{i}-\overline{B V F_{z}}\right|\right)
$$

subject to

$$
\begin{gathered}
g\left(x_{1}, x_{2}, \ldots, x_{n}\right)=H_{\text {calculate }}-H_{\text {desired }} \leq 0.0 \\
x_{1} \in A_{1} \\
x_{2} \in A_{2} \\
\vdots \\
x_{n} \in A_{n},
\end{gathered}
$$

where variables $x_{n}$ represent the above-mentioned parameters, $A_{n}$ represents the variable bounds, $H_{\text {calculate }}$ represents the estimated head, and $H_{\text {desired }}$ represents the desired head. The meaning and its bounds of each variable are listed in Table 1, and their bounds are used to design an impeller with $200 \mathrm{~m}^{3} / \mathrm{h}$ capacity and $20 \mathrm{~m}$ head. Table 2 summarizes the settings that have been used for the GA.

In order to make sure of the availability and advantage of this method, another 5 impellers are designed by using single arc (Model 1), double arcs (Model 2), triple arcs (Model 3), logarithmic spiral (Model 4), and linear-variable angle spiral (Model 5) as blade profiles with the same design parameters 
TABLE 1: Optimal variables.

\begin{tabular}{llc}
\hline Variable & & Bounds \\
\hline$x_{1}$ & Meridional flow factor & $(0.0,1.0)$ \\
$x_{2}$ & Incidence angle & $(3.0,15.0)$ \\
$x_{3}$ & Deviation angle & $(1.0,5.0)$ \\
$x_{4}$ & Wrapping angle & $(80.0,160.0)$ \\
$x_{5}$ & Relative velocity distribution factor & $(-1.0,1.0)$ \\
$x_{6}$ & Bezier control point 1 on hub & $(0.0,1.0)$ \\
$x_{7}$ & Bezier control point 2 on hub & $(0.0,1.0)$ \\
$x_{8}$ & Bezier control point 3 on shroud & $(0.0,1.0)$ \\
$x_{9}$ & Bezier control point 4 on shroud & $(0.0,1.0)$ \\
\hline
\end{tabular}

determined by empirical correlations. These parameters are listed in Table 3.

For the single arc design Model 1, only one arc curve is used to form the blade profile which results in the blade angle along the radial direction increasing at first till almost $1 / 3$ radius and then decreasing till trailing edge, as shown in Figure 6. This type of blade angle distribution may lead to flow separation on blade surface and eventually bring detrimental effect on the performance. For the double arcs design Model 2 , the blade angle changing rate is less than Model 1 and its distribution is formed by two convex curves. And the blade angle distribution of triple arcs design Model 3 is formed by three convex curves. It can be concluded that the blade profile formed with more arcs will have less angle changing rate and may have better performance. Thus, the other two types of impellers have been designed, one type of which is with constant blade angle and the other with linear changing. These two impellers are logarithmic spiral type and variable angle spiral type. For these two, blade angle will fulfill the following expression at any points on profile line:

$$
\tan \beta=\frac{d r}{r \cdot d \theta}
$$

For logarithmic spiral (Model 4), $\beta=$ Const.

For linear-variable angle spiral (Model 5), $\beta=a \cdot \theta+b$.

As it can be seen from Figure 7, the blade wrapping angles of Model 4 and Model 5 are larger than Model 1, Model 2, and Model 3. And the impeller with more arcs used in profile will be with larger wrapping angles.

\section{Numerical Simulation by CFD}

At present by using numerical procedures, it is possible to predict the performance curves of impellers with enough accuracy. CFD (computational fluid dynamics) analysis is currently being widely used in design procedures to reduce prototypes testing for centrifugal impellers. For study of the hydraulic performance of these 6 impellers, the governing equations of these flow fields in impellers are solved by commercial CFD code FLUENT to predict three-dimensional turbulent fluid flow.

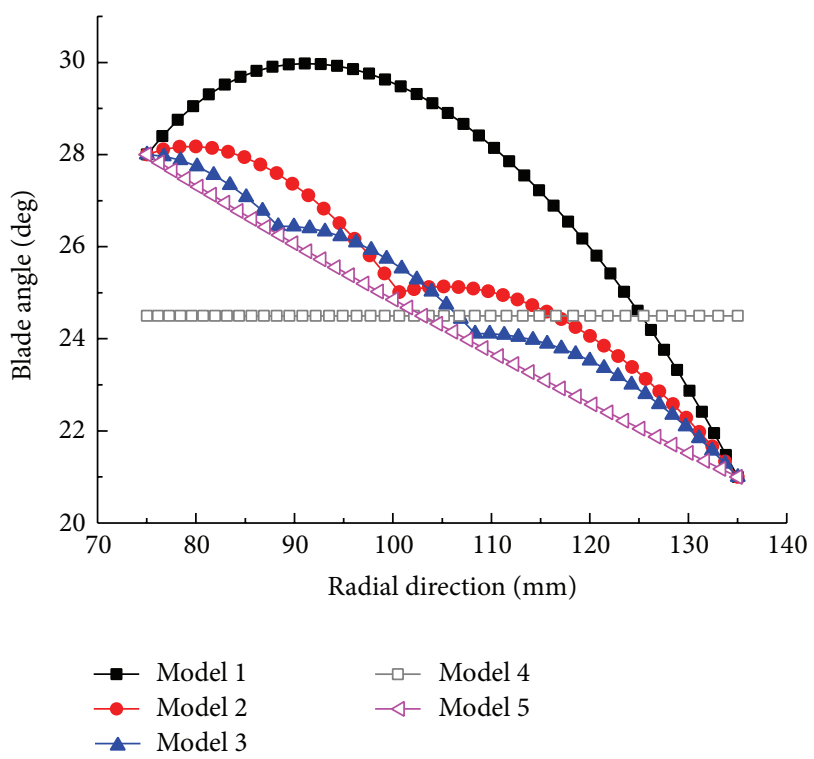

FIGURE 6: Blade angle distribution along the radial direction of 5 impeller blades.

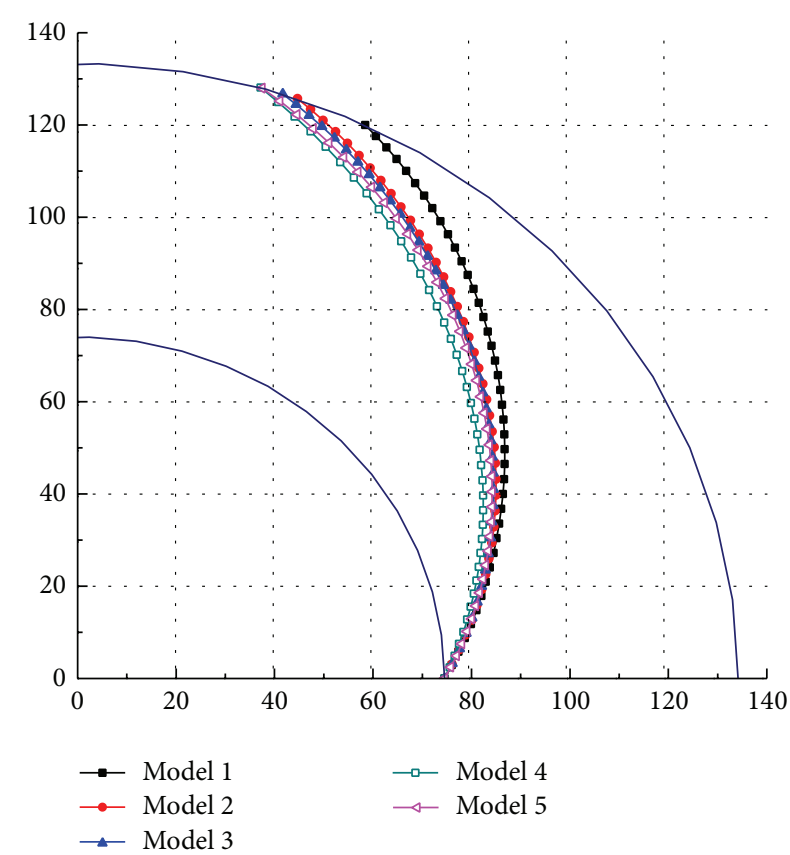

Figure 7: The camber line profiles of 5 impeller blades.

3.1. Geometry and Grid. The 3D solid geometry models of 6 designed impellers have been generated, as shown in Figure 8. It can be clearly seen that the optimal design impeller has the largest wrapping angle and the narrowest flow channels, which make the blades have a better control on the fluid to prevent flow separations.

The calculation domain of centrifugal pump is mainly composed of inlet extension, impeller, volute, and outlet extension. Reasonable lengths of inlet and outlet extensions are added to the real machine geometry to reduce the 
TABle 2: Settings for the GA.

\begin{tabular}{llccccc}
\hline Populations size & Initial population & Generations & Elite members & Crossover rate & Mutation rate & Selection method \\
\hline 300 & Uniform random & 100 & 6 & 0.6 & 0.01 & Roulette wheel \\
\hline
\end{tabular}

TABLE 3: Design parameters.

\begin{tabular}{lcccccc}
\hline$D_{1}(\mathrm{~mm})$ & $D_{2}(\mathrm{~mm})$ & $B_{2}(\mathrm{~mm})$ & $\beta_{1}$ (degree) & $\beta_{2}$ (degree) & Zn & Rotating speed (rpm) \\
\hline 150 & 270 & 30 & 28 & 21 & 6 & 1450 \\
\hline
\end{tabular}

unavoidable effect of inlet and outlet boundaries on the final flow solution as a result of the boundary conditions, as shown in Figure 9(a). The discretization of the calculation domain is done keeping the balance between calculation time and the accuracy order of the simulation of the flow structure. Unstructured hexahedra and tetrahedral grid with strong flexibility are used to define the impeller domains. Special care should be taken in the regions near the blades. Once the geometries are defined and grids are ready, these models are ready to be simulated. The grids generated for one of these 6 impellers are shown in Figure 9(b).

3.2. Boundary Conditions. The modeled boundary conditions are those considered most physically meaningful for flow simulations and those that give a flow solution restricted by them. The type of inlet boundary condition is velocity inlet assumed to be a uniform velocity distribution at this plane. The magnitudes of the velocity vectors in radial and tangential directions are assumed to be zero, while axis direction velocity is assumed to be a uniform velocity distribution and is computed from the specified mass flow rate. For outlet, the outflow is given as boundary condition. Interface pairs are set between the stationary and rotational regions. The solid walls such as blade surfaces, hub, and shroud are given the moving wall and others are given the stationary wall. Nonslip conditions have been imposed over all wall boundaries. The operation condition of impeller (flow rate) can be changed through modifying the axis velocity magnitude at inlet boundary. Water is used as working fluid in ambient condition.

3.3. Flow Calculation. FLUENT software is adopted to solve the fully $3 \mathrm{D}$ incompressible Navier-Stokes equations to simulate the inner flow field by assuming that the fluid is steady, viscous, and incompressible. Multiple reference frame (MRF) model is applied to take into account the interaction between stationary volute and rotating impeller. The RNG $k-\varepsilon$ turbulence is activated to handle the turbulence effects. Wall functions, based on the logarithmic law, have been used to estimate wall shear stress and pressure. The pressure-velocity coupling is calculated through the SIMPLEC algorithm and the pressure correction under-relaxation factor is set to 1.0. Second order, upwind discretization has been used for convection terms and PRESTO! Scheme has been used for pressure terms.

3.4. Grid Independence. For the reduction of computation time and the improvement of accuracy, the optimum number of grid cells of the optimal designed pump in the simulation has been investigated. In Table 4, it is observed how the head coefficient and hydraulic efficiency reach an asymptotic value as the number of cells increases. Balance between calculation time and the accuracy order of the simulation has been made and the grid with cell number 1129299 is considered to be sufficiently reliable. By using the same procedures, grids for the other 5 impellers are also decided (Model 1 cell number: 1091070, Model 2 cell number: 1086338, Model 3 cell number: 1134753, Model 4 cell number: 1200932, and Model 5 cell number: 105985).

\section{Simulation Results Analysis}

The flows in these 6 designed impellers passages with the same volute have been simulated at the design point $(Q=$ $200 \mathrm{~m}^{3} / \mathrm{h}$ ). The rotation of the impeller is in the anticlockwise direction.

Firstly, averaged fields are studied in order to evaluate the overall treads. The static pressure contours are displayed in Figure 10 on the middle-span plane and in Figure 11 on blade pressure surfaces of these 6 impellers for viscous fluid flow, respectively. As the fluid moves along the passage, the pressure increases and there are some very low pressure regions on suction sides of blades near the leading edges in Model 1 to Model 5, except Model 6 (optimal designed impeller), on which nearly no low pressure regions exist. Among these 5 impellers, the largest low pressure region exists in Model 1. These low pressure regions will have undesirable effects on impeller's performance, especially when the pressure value is lower than evaporation pressure under that condition. The low pressure regions may be mainly caused by incidence loss due to mismatch of hub and the position of leading edge. Compared to other impellers, the pressure increasing along the flow channel is more preferable and the pressure distribution around the exit of impeller is shown to be more uniform, which will cause less loss.

The relative velocity streamlines are displayed in Figure 12 on the middle-span plane of these 6 impellers for viscous fluid flow, respectively. It can be clearly seen that there is an axial vortex in impeller channel near suction surface of blade of Model 1, Model 2, and Model 5, respectively, which means that flow separation happens on this region. This will reduce the flow area which may bring about more losses because of the rising flow velocity and will also cause energy losses due to viscosity of flow medium. Among these 3 impellers, the largest vortex exists in Model 1, which is very harmful to its performance. These may be caused by the improper 


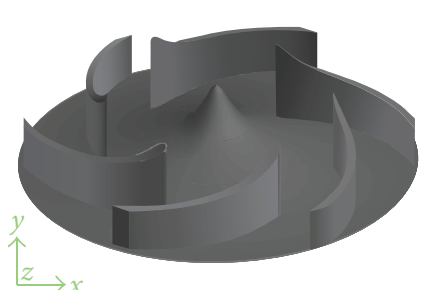

(a) Model 1

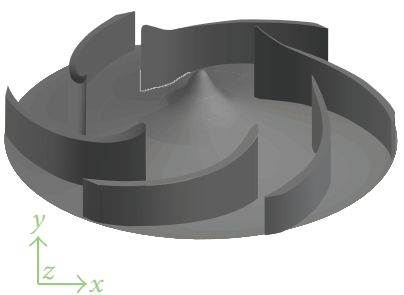

(c) Model 3

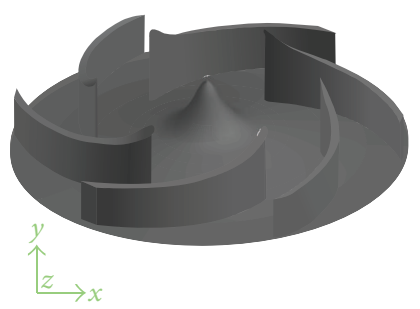

(e) Model 5

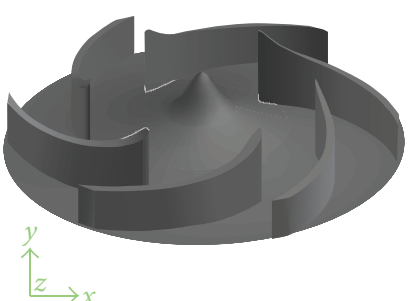

(b) Model 2

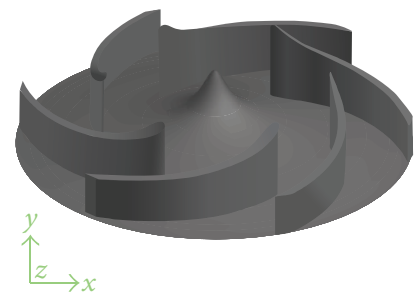

(d) Model 4

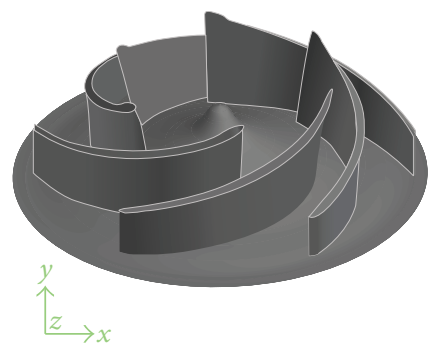

(f) Optimal design

FIGURE 8: Geometry models of impellers.

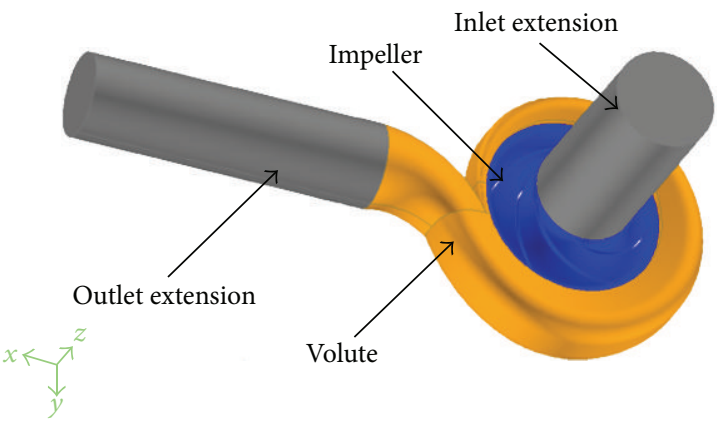

(a) Computational domain

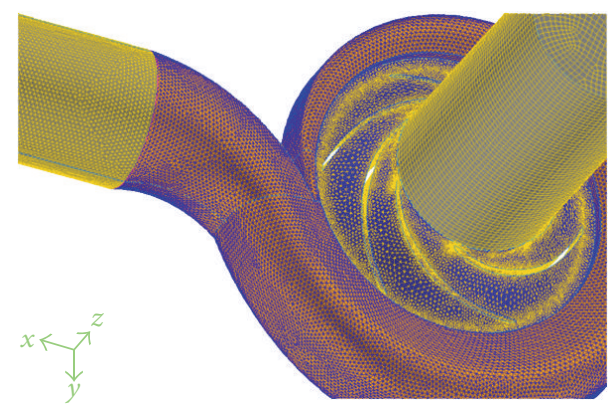

(b) Grid of the domain

FIGURE 9: Computational domain and grid.

blade angle distribution; the separation point of Model 1 is around $3 / 4$ radius, at which point the blade angle given is about 29 degrees. For Model 2, the separation point is around $3 / 5$ radius, at which point the blade angle given is about 28 degrees, and for Model 5, the separation point is nearly the leading edge point.

With the purpose to use the advantage of localizing diagnosis of performance of these 6 different impellers, the BVF analysis is introduced. Figure 13 shows the distribution of $\mathrm{BVF}_{z}$ (only $\sigma_{P z}$ component, which is important on moment generation) and its negative values distribution (the lower part, in which the magnitude of negative $\mathrm{BVF}_{z}$ is used). These distributions are obtained by calculating the expression $\sigma_{P z}=$ $\boldsymbol{\sigma}_{P} \cdot \mathbf{e}_{z}$, after CFD simulation.

It can be seen Figure 13 (from Model 1 to Model 5) that these are positive $\mathrm{BVF}_{z}$ regions near both leading edges and trailing edges of blades. These regions are unfavorable to transfer the energy to fluid, especially the region near trailing edges, since the product of negative $\mathrm{BVF}_{z}$ and the square of $r$ (the radial position) contributes to generate positive moment. Among these 6 impellers, the largest negative $\mathrm{BVF}_{z}$ value exists in Model 6 (optimal design) around the 
TABLE 4: Grid independence analysis.

\begin{tabular}{lccccc}
\hline Cell number & 625173 & 841281 & 1129299 & 1358154 & 1543701 \\
\hline Head coefficient & 0.836 & 0.843 & 0.849 & 0.853 & 0.856 \\
Efficiency & 0.7573 & 0.7698 & 0.7743 & 0.7763 & 0.7780 \\
\hline
\end{tabular}

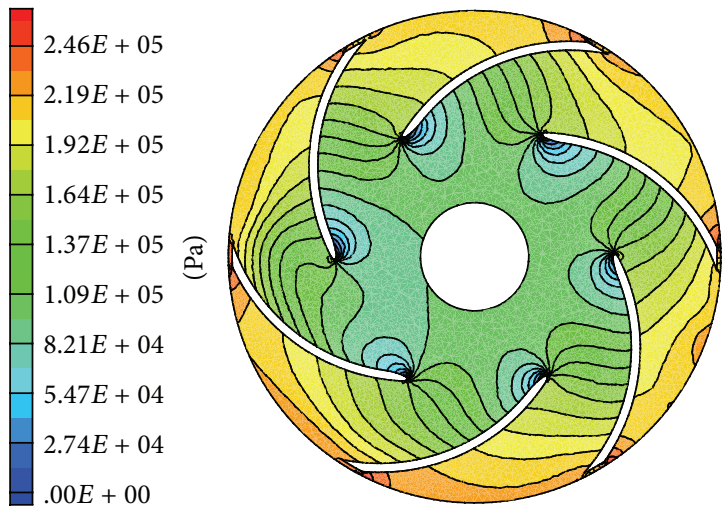

(a) Model 1

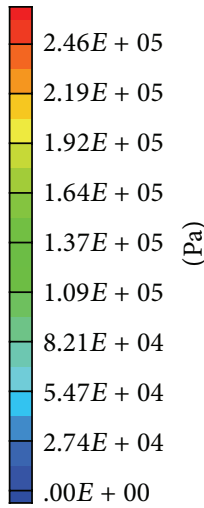

$2.46 E+05$
$2.19 E+05$
$1.92 E+05$
$1.64 E+05$
$1.37 E+05$ ह
$1.09 E+05$
$8.21 E+04$
$5.47 E+04$
$2.74 E+04$
$.00 E+00$

(c) Model 3

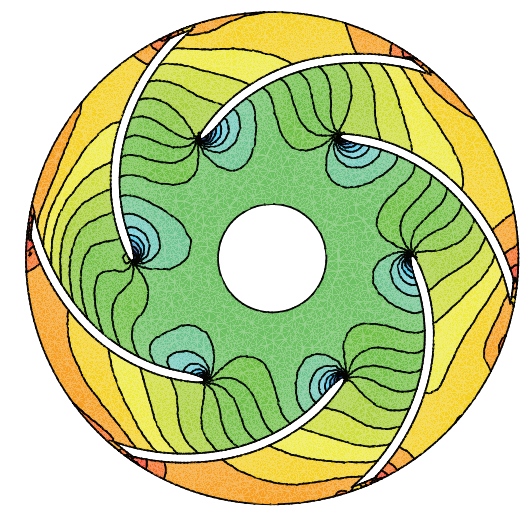

(e) Model 5

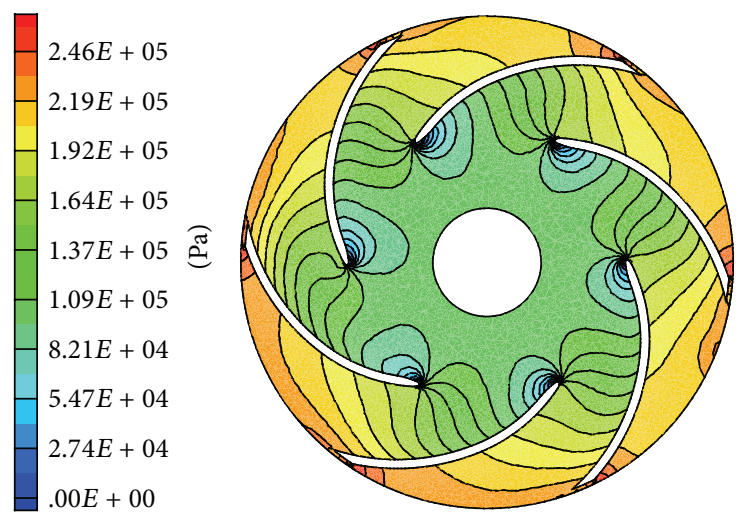

(b) Model 2

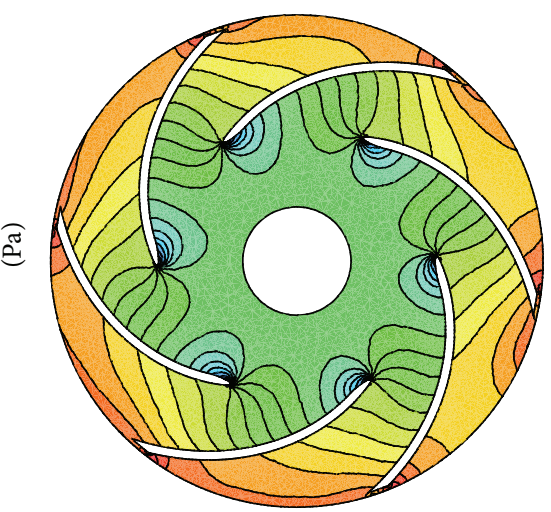

(d) Model 4

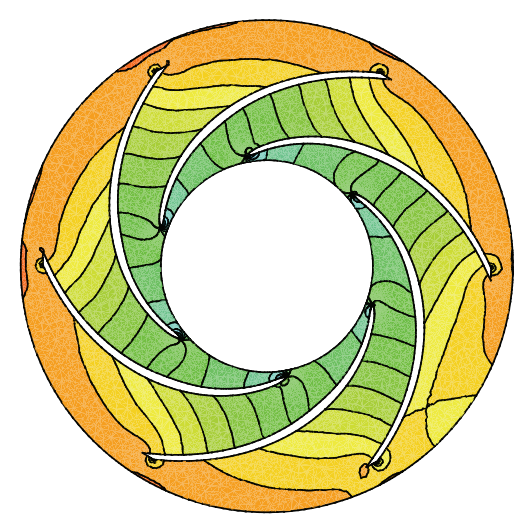

(f) Optimal design

FIGURE 10: Static pressure distribution.

trailing edge region. As it also can be seen from these $\mathrm{BVF}_{z}$ distribution figures, separation points in the blade surfaces of these impellers where flow separations occur are nearly at the same position on the blades, where low $\mathrm{BVF}_{z}$ values exist. And compared to other impellers, the distribution of
$\mathrm{BVF}_{z}$ on optimal impeller blade surface is more uniform, which can reflect that the GA optimal design method is available and can be used to solve the desired object function optimization problem and the estimated $\mathrm{BVF}_{z}$ calculation is feasible. But the negative region is a little small which will 


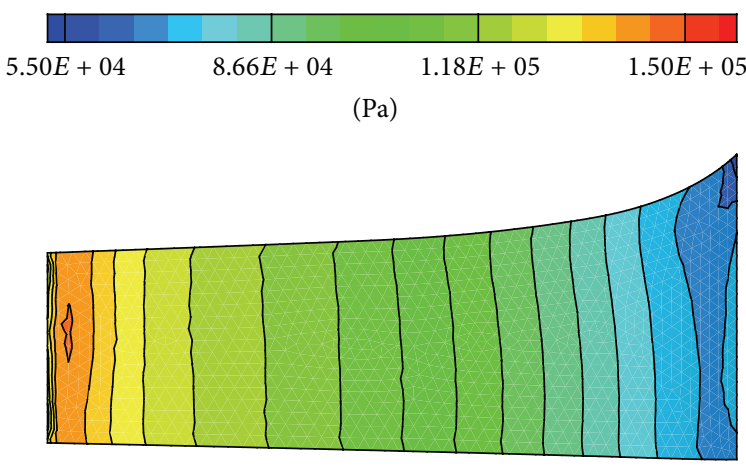

(a) Model 1

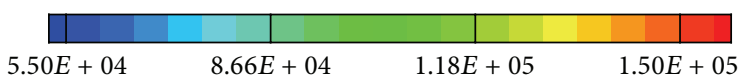

$(\mathrm{Pa})$

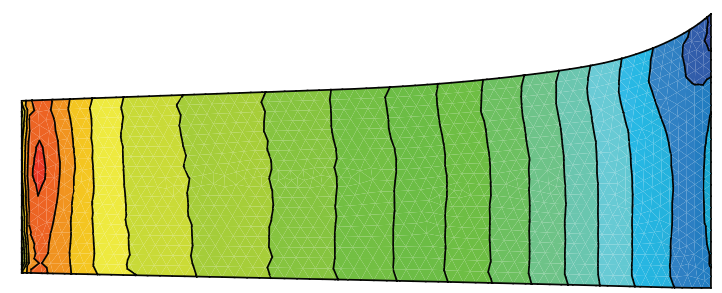

(c) Model 3

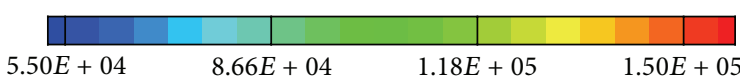

$(\mathrm{Pa})$

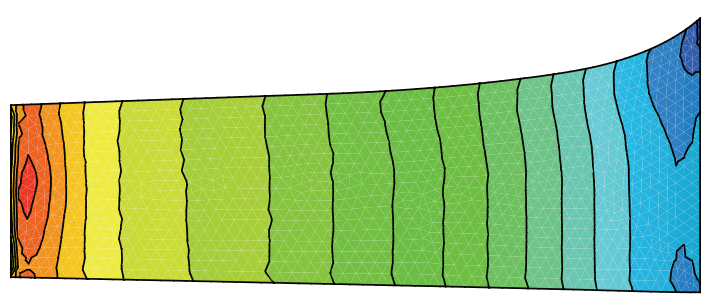

(e) Model 5

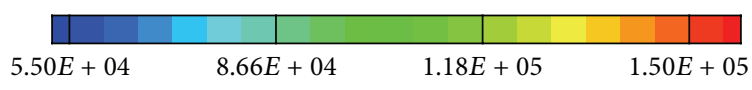

$(\mathrm{Pa})$

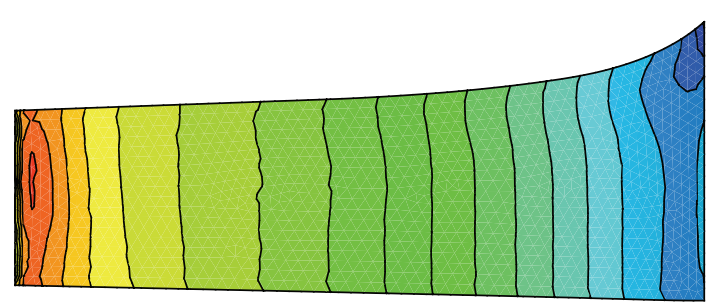

(b) Model 2

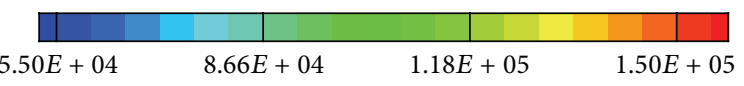

$(\mathrm{Pa})$

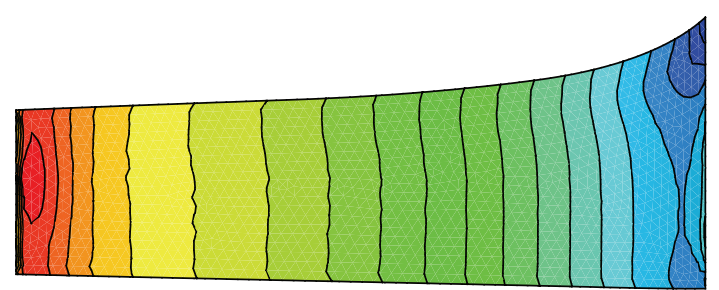

(d) Model 4

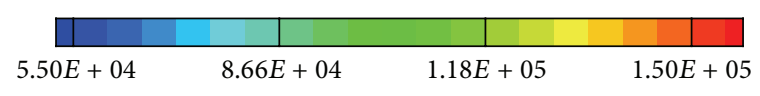

$(\mathrm{Pa})$

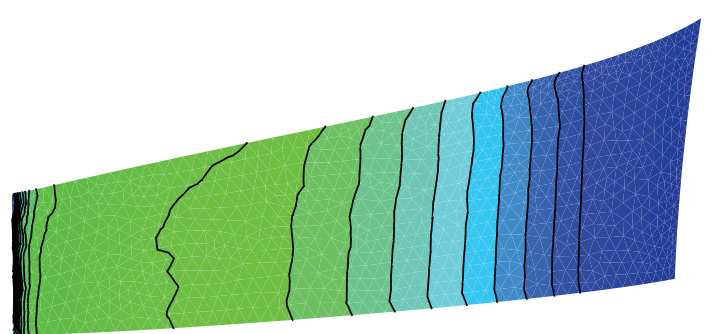

(f) Optimal design

FIGURE 11: Static pressure distribution on blade's pressure sides.

result in insufficient moment transfer. This may be due to the improper head estimation lack of enough consideration about the viscous loss and other loss.

In order to compare the hydraulic performances of these impellers, the head and hydraulic efficiency are estimated using the following expression. The head $H$ gained by the fluid through the impeller is computed from the total energy of the fluid at the inlet and outlet of the impeller:

$$
H=H_{2}-H_{1}=\frac{1}{Q} \cdot \int\left(\frac{p_{2}-p_{1}}{\rho g}+\frac{c_{2}^{2}-c_{1}^{2}}{2 g}\right) \cdot d q,
$$

where the right-hand side integral can be approximated by a summation over the radial flow rates $d q$ at all grid cells facing the inlet or outlet of the impeller.

The hydraulic efficiency of the impeller is defined as the ratio of the net head $(H)$ added to the passing fluid, divided by the energy $\left(H_{u}\right)$ given at the impeller shaft. And $H_{u}$ can be calculated from the torque $M_{u}$ developed on the blades

$$
H_{u}=\frac{\omega_{r} \cdot M_{u}}{\rho \cdot g \cdot Q}=\frac{\omega_{r} \cdot \int\left[(\mathbf{r} \times \mathbf{n})+\left(\mathbf{r} \times \boldsymbol{\tau}_{w}\right) \cdot \cot \beta\right] \cdot b \cdot d r}{\rho \cdot g \cdot Q} .
$$

So the hydraulic efficiency of the impeller can be finally defined as

$$
\eta=\frac{H}{H_{u}} .
$$

Nondimensional characteristics (head coefficient) that are basically derived from the similarity of centrifugal pumps are used [18]:

$$
\text { Head Coefficient }=\frac{H}{u_{2}^{2} / 2 g} \text {. }
$$




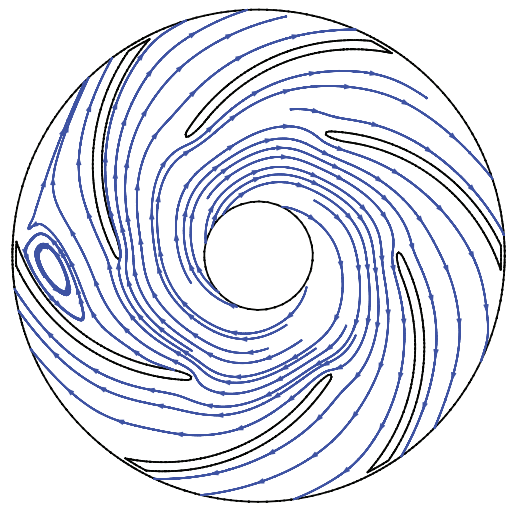

(a) Model 1

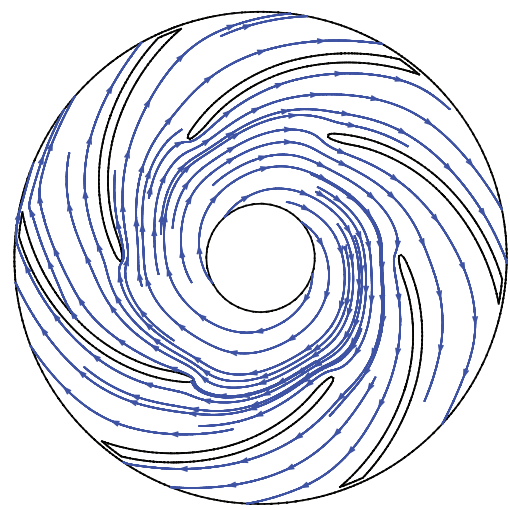

(c) Model 3

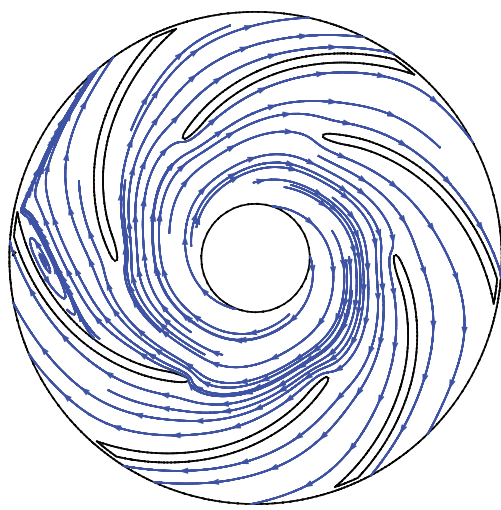

(e) Model 5

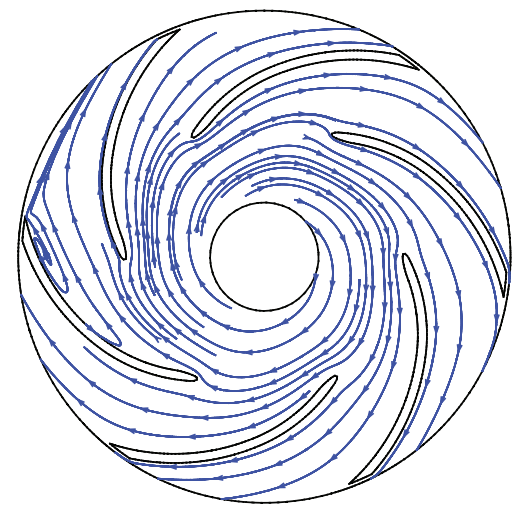

(b) Model 2

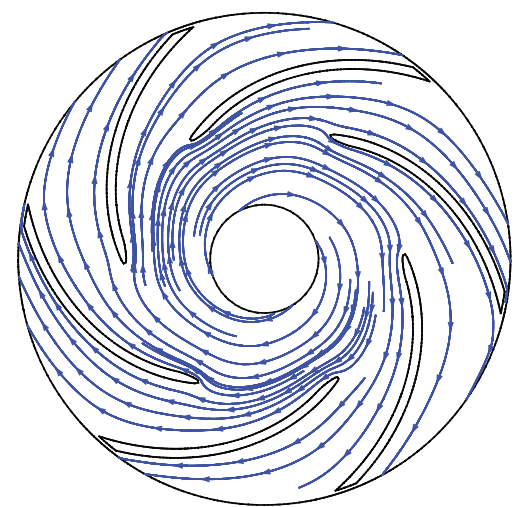

(d) Model 4

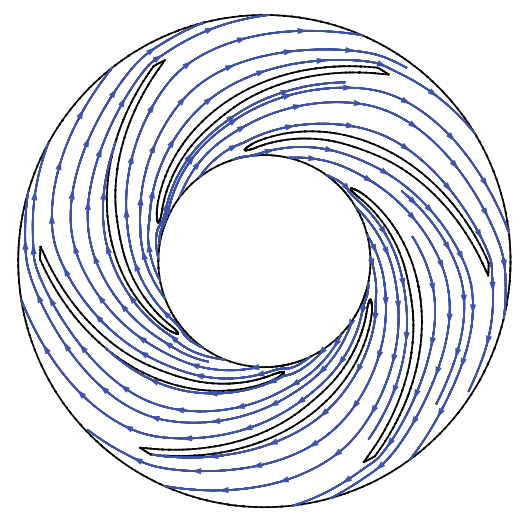

(f) Optimal design

FIGURE 12: Streamline.

TABLE 5: The estimated performance of 6 impellers at design point.

\begin{tabular}{lcccccc}
\hline & Model 1 & Model 2 & Model 3 & Model 4 & Model 5 & Optimal design \\
\hline Head coefficient & 0.849 & 0.838 & 0.836 & 0.876 & 0.834 & 0.820 \\
Efficiency & $77.4 \%$ & $78.1 \%$ & $78.4 \%$ & $80.9 \%$ & $78.6 \%$ & $87.5 \%$ \\
\hline
\end{tabular}

The head coefficients and hydraulic efficiency of these 6 impellers at design point are listed in Table 5. The optimal design impeller is with the maximal efficiency and Model 1 is with the minimal efficiency, which is consistent with the above statements. Model 4 is with the maximal head coefficient and optimal design with the minimal one, but compared to Model 1, Model 2, Model 3, and Model 5, the optimal impeller has much better hydraulic performance at the cost of only maximal $3.4 \%$ head loss.

\section{Conclusion}

Based on two-dimensional theory, boundary vorticity flux diagnosis, and global search technique, a GA optimal 


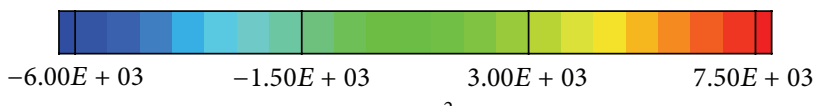
$\left(\mathrm{m} \cdot \mathrm{s}^{-2}\right)$
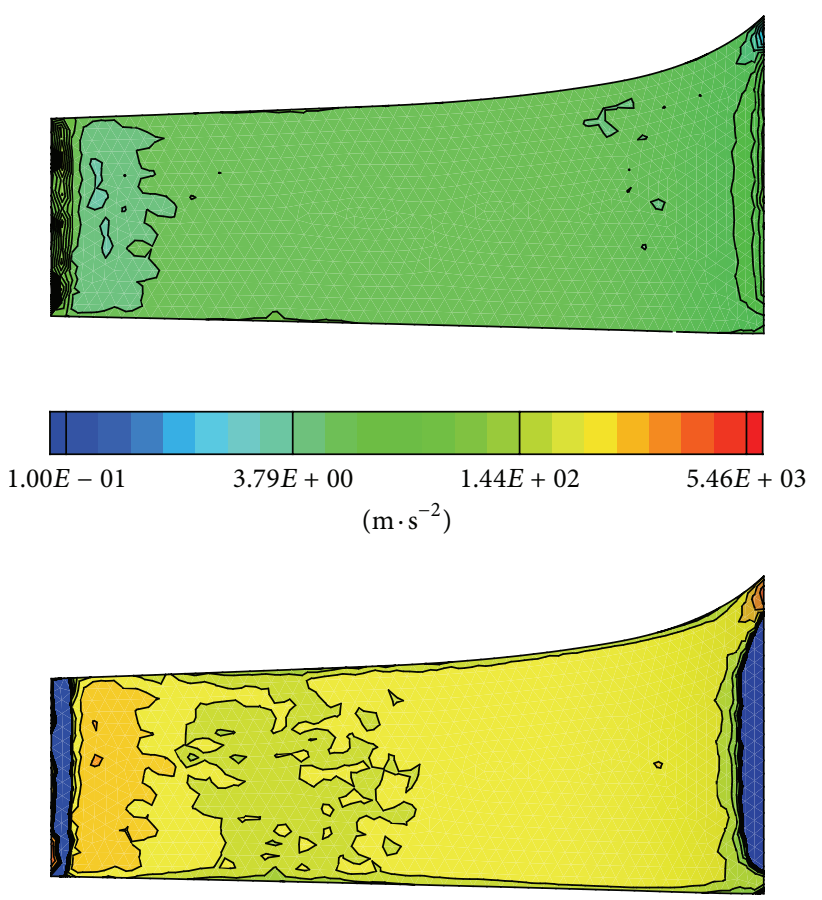

(a) Model 1

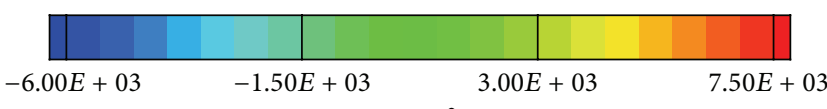
$\left(\mathrm{m} \cdot \mathrm{s}^{-2}\right)$
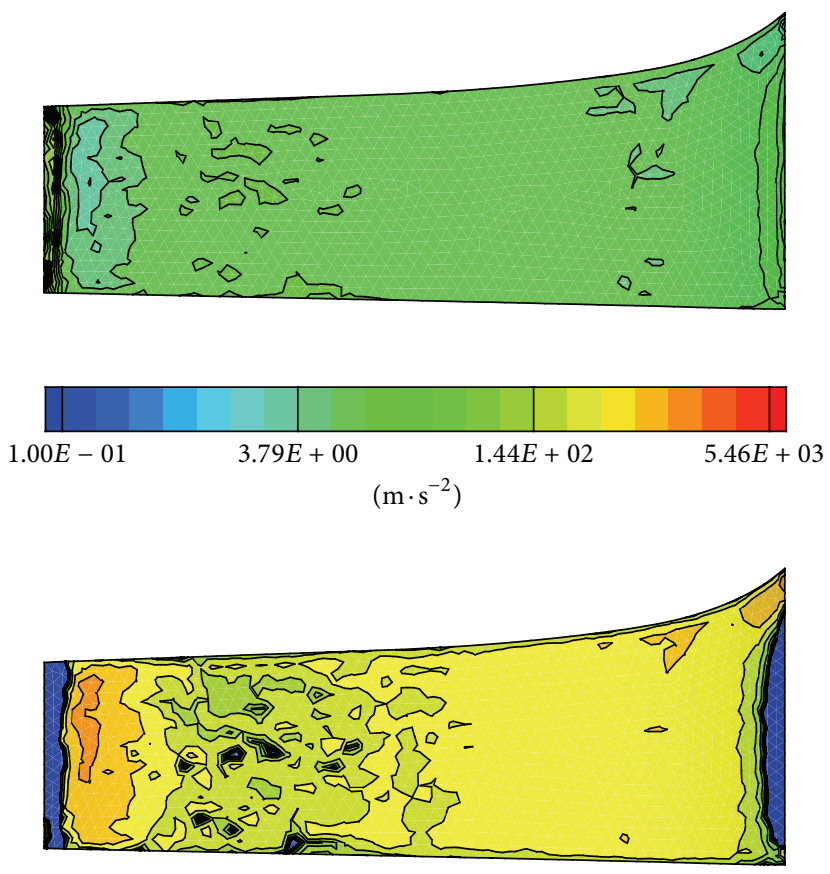

(c) Model 3
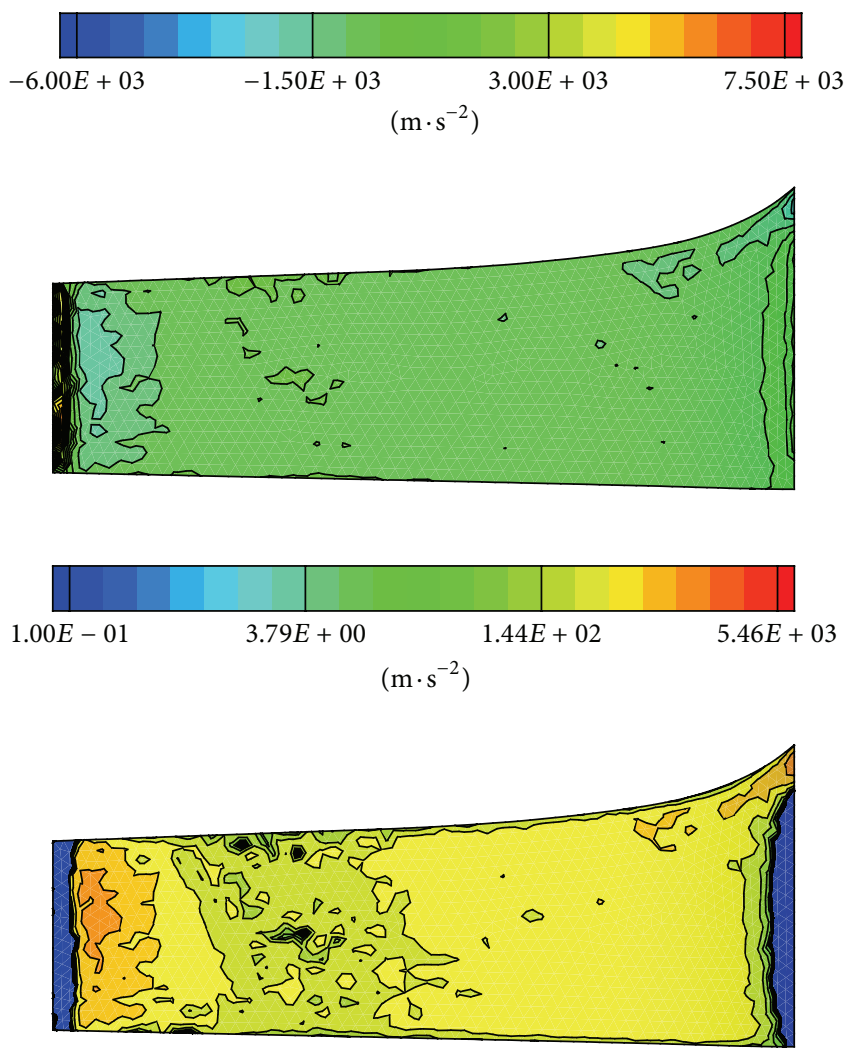

(b) Model 2

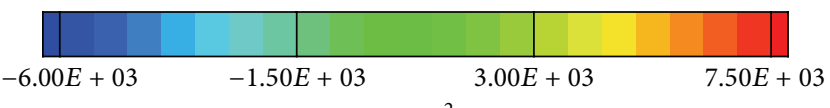
$\left(\mathrm{m} \cdot \mathrm{s}^{-2}\right)$
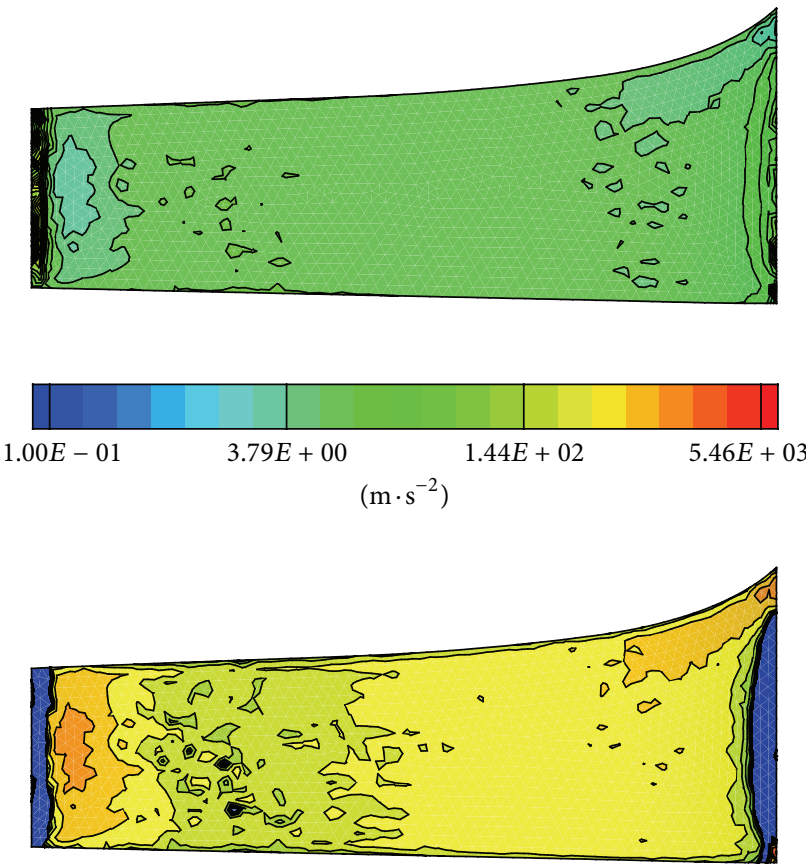

(d) Model 4

Figure 13: Continued. 

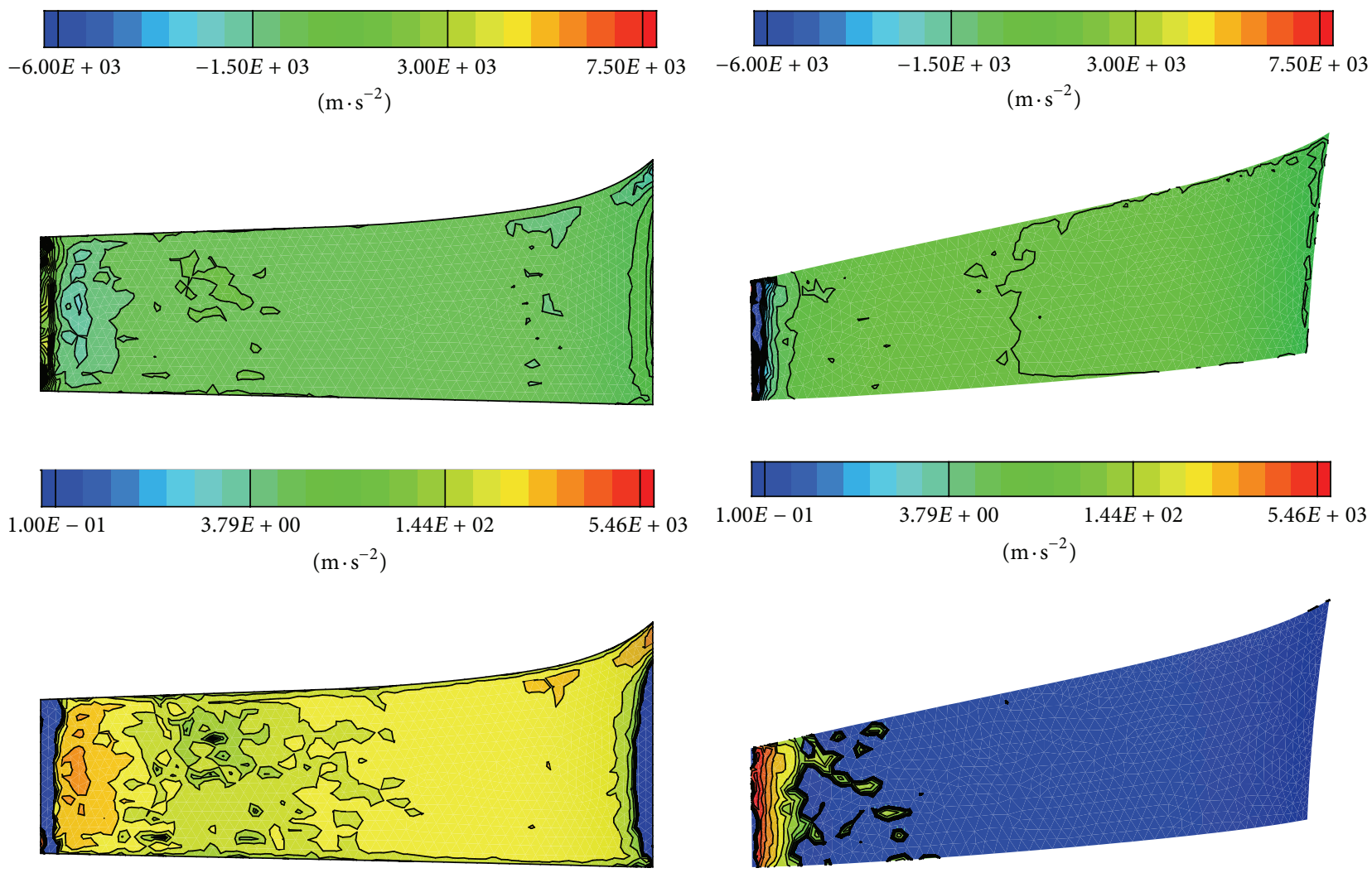

(e) Model 5

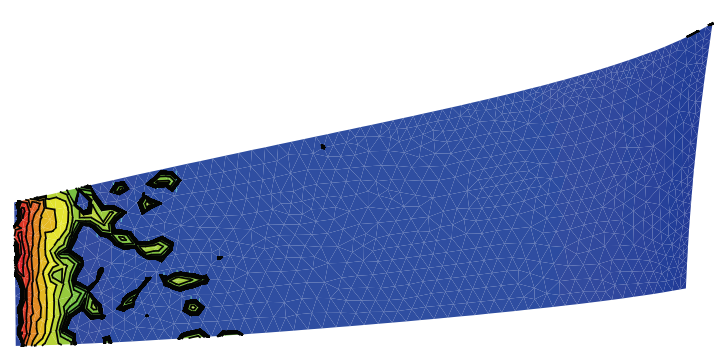

(f) Optimal design

FIGURE 13: BVF $_{z}$ distribution on blade's pressure sides.

hydraulic design method is developed. In this method, the estimated boundary vorticity flux values on blade surface are used in the evaluation function of genetic algorithm. Another 5 impellers have been designed as comparison and 3D numerical simulation is adopted to investigate the flow field inside the impellers and the hydraulic performances of them. The results show that the hydraulic performance of impeller designed by this method is much better than the other 5 impellers under design operation condition, with almost the same head, higher efficiency, and lower rotating torque, which means less hydraulic loss and energy consumption. Uniform $\mathrm{BVF}_{z}$ distribution on the blade surface of optimal designed impeller confirms that the desired object function optimization is realized and this optimal hydraulic design method is available. But it may be due to an insufficient consideration on head estimation to care about all losses; the head of optimal designed impeller is not as good as expected. Further works are needed to realize a better head estimation or to introduce some more constraint conditions in the optimal model.

\section{Nomenclature}

$C_{m}:$ Meridional velocity

$W$ : Relative velocity

$U$ : Peripheral velocity

c: Absolute velocity

$\omega_{r}$ : Angle velocity
$D_{1}$ : Impeller diameter at inlet

$D_{2}$ : Impeller diameter at outlet

$B_{2}$ : Impeller width at outlet

$\beta_{1}$ : Inlet blade angle

$\beta_{2}$ : Outlet blade angle

$\varphi$ : Wrapping angle

$\theta$ : Angular direction

n: Rotation speed

Q: Flow rate

$u_{2}$ : Peripheral velocity at impeller outlet

$Z n$ : Blade number

$\rho: \quad$ Density of the fluid

$M_{u}$ : Torque

$H$ : Head

$\eta$ : Efficiency

$\sigma:$ Boundary vorticity flux

$P$ : Pressure

$\boldsymbol{\tau}_{w}:$ Wall shear stress

$\alpha_{1}$ : Angle between meridional streamline and vertical line

$\gamma$ : Angle between quasiorthogonal line and axis

$\psi$ : Expelling coefficient

$l$ : Length of meridional streamline

$s$ : Length of quasiorthogonal line

$r$ : Radial direction

$\delta$ : Angle between normal line of quasiorthogonal line and meridional streamline. 


\section{Conflict of Interests}

The authors declare that there is no conflict of interests regarding the publication of this paper.

\section{References}

[1] A. Goto, M. Nohmi, T. Sakurai, and Y. Sogawa, "Hydrodynamic design system for pumps based on 3-D CAD, CFD, and inverse design method," Journal of Fluids Engineering, vol. 124, no. 2, pp. 329-335, 2002.

[2] V. A. Grapsas and S. John, "Anagnostopoulos, Dimitrios E. Papantonis. Hydrodynamic design of radial flow pump impeller by surface parameterization," in Proceedings of the 1st International Conference on Experiments/Process/SystemModelling/Simulation/Optimization, 2005.

[3] S. Derakhshan, B. Mohammadi, and A. Nourbakhsh, "The comparison of incomplete sensitivities and Genetic algorithms applications in 3D radial turbomachinery blade optimization," Computers and Fluids, vol. 39, no. 10, pp. 2022-2029, 2010.

[4] J. S. Zhou, S. J. Zhang, and P. Z. Mao, "Performance optimization based on genetic algorithm of double suction centrifugal pump," Advanced Materials Research, vol. 468, pp. 2565-2568, 2012.

[5] D. S. Li, W. W. Song, J. Fu et al., "Applied micro genetic algorithm to optimize design low specific speed pump impeller," Advanced Materials Research, vol. 816, pp. 1010-1014, 2013.

[6] J. Z. Wu and J. M. Wu, "Vorticity dynamics on boundaries," Advances in Applied Mechanics, vol. 32, pp. 119-275, 1996.

[7] J. Z. Wu and J. M. Wu, "Boundary vorticity dynamics since Lighthill's 1963 article: review and development," Theoretical and Computational Fluid Dynamics, vol. 10, no. 1-4, pp. 459474, 1998.

[8] J. Z. Wu, H. Y. Ma, and M. D. Zhou, Vorticity and Vortex Dynamics, Springer, Berlin, Germany, 2006.

[9] Z. J. Wu, L. R. Roach, F. C. Lo et al., "Aerodynamic diagnostics and design based on boundary vorticity dynamics," in Proceedings of American Institute of Aeronautics and Astronautics Conference (AIAA '99), pp. 33-46, American Institute of Aeronautics and Astronautics, Norfolk, Va, USA, 1999.

[10] Y. Zhang, X. Luo, H. Ding, X. Wang, and H. Xu, "Design optimization of the impeller for a high specific-speed pump based on BVF diagnosis," Journal of Engineering Thermophysics, vol. 31, no. 5, pp. 765-768, 2010.

[11] J. Z. Wu, X. Y. Lu, Y. T. Yang et al., "Vorticity dynimics in complex flow diagnosis and management. P.Y. Chou Memorial Lecture," in Proceedings of the 13th Asian Congress of Fluid Mechanics, Dhaka, Bangladesh, December 2010.

[12] Y. Z. Zhang, L. Chen, X. Zhou et al., "Hydraulic design, numerical simulation and BVF diagnosis of high efficiency centrifugal pump," IOP Conference Series: Earth and Environmental ScienceEmail alert RSS feed, vol. 15, Article ID 032024, 2012.

[13] S. Kaewnai, M. Chamaoot, and S. Wongwises, "Predicting performance of radial flow type impeller of centrifugal pump using CFD," Journal of Mechanical Science and Technology, vol. 23, no. 6, pp. 1620-1627, 2009.

[14] M. Nataraj and R. Ragoth Singh, "Analyzing pump impeller for performance evaluation using RSM and CFD," Desalination and Water Treatment, 2013.

[15] Z. Wang, J. Zheng, L. Li, and S. Luo, "Research on threedimensional unsteady turbulent flow in multistage centrifugal pump and performance prediction based on CFD," Mathematical Problems in Engineering, vol. 2013, Article ID 589161, 7 pages, 2013.

[16] Y. Zhang, X. Zhou, Z. Ji, and C. Jiang, "Numerical design and performance prediction of low specific speed centrifugal pump impeller," Journal of Engineering Thermophysics, vol. 31, pp. 1720, 2010.

[17] X. Zhou, Y. X. Zhang, Z. L. Ji, and L. Chen, "Hydraulic design and performance analysis of low specific speed centrifugal pump," IOP Conference Series: Earth and Environmental Science, vol. 15, Article ID 032023, 2012.

[18] P. Girdhar and O. Moniz, Practical Centrifugal Pumps, Elsevier, 2005. 

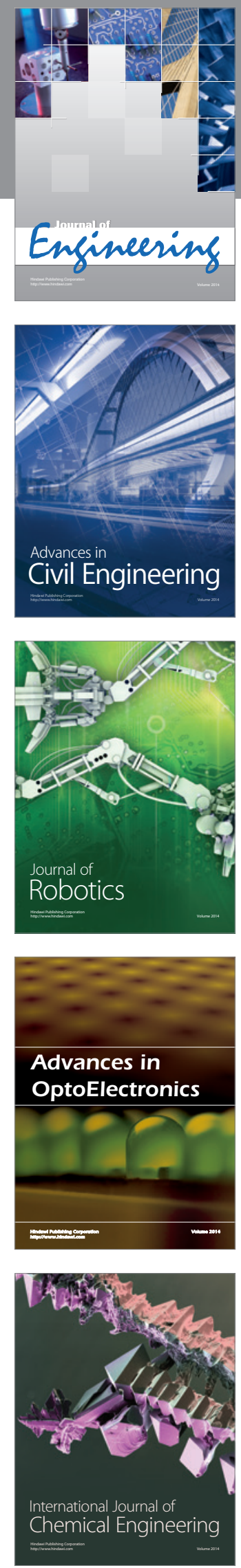

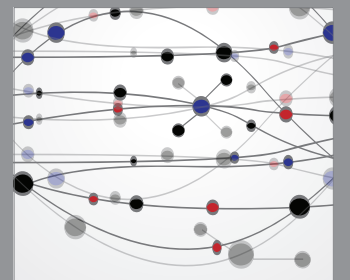

The Scientific World Journal
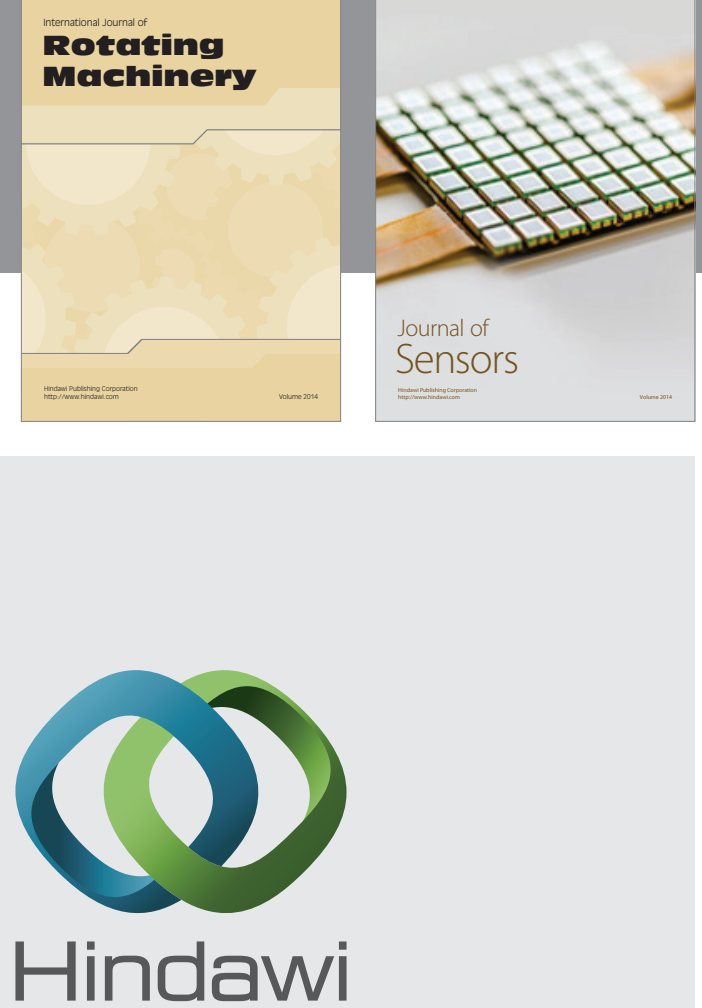

Submit your manuscripts at http://www.hindawi.com
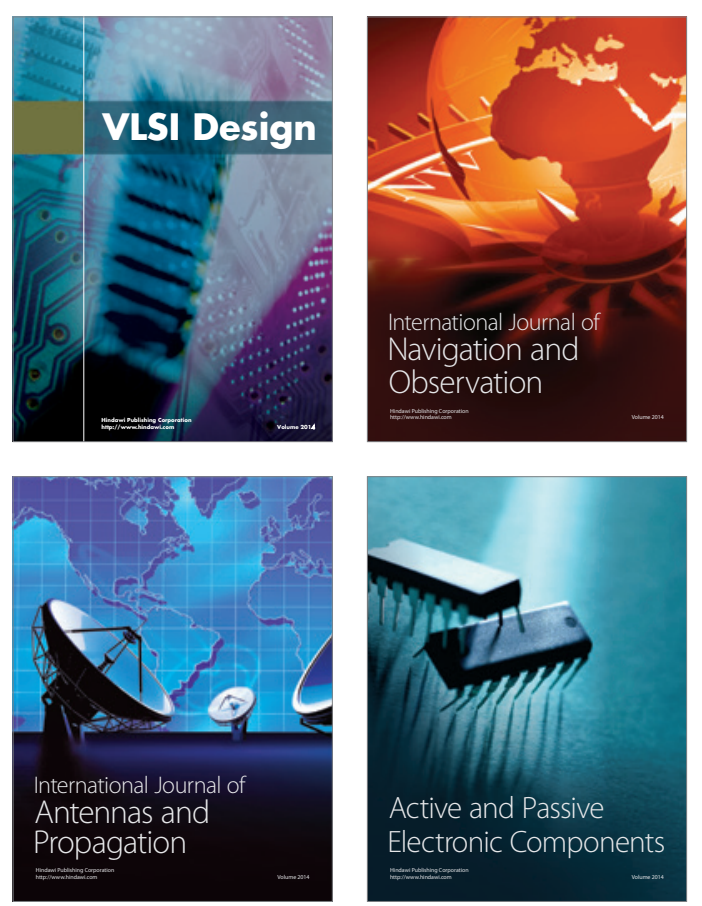
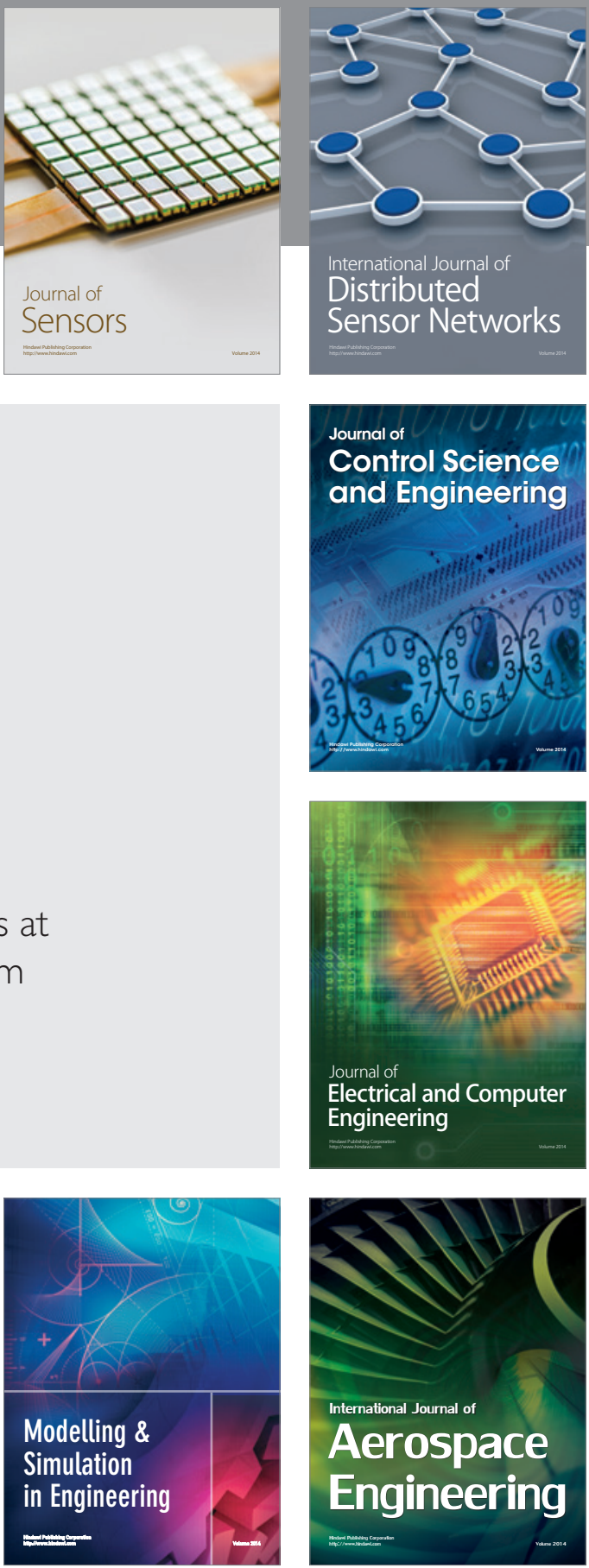

Journal of

Control Science

and Engineering
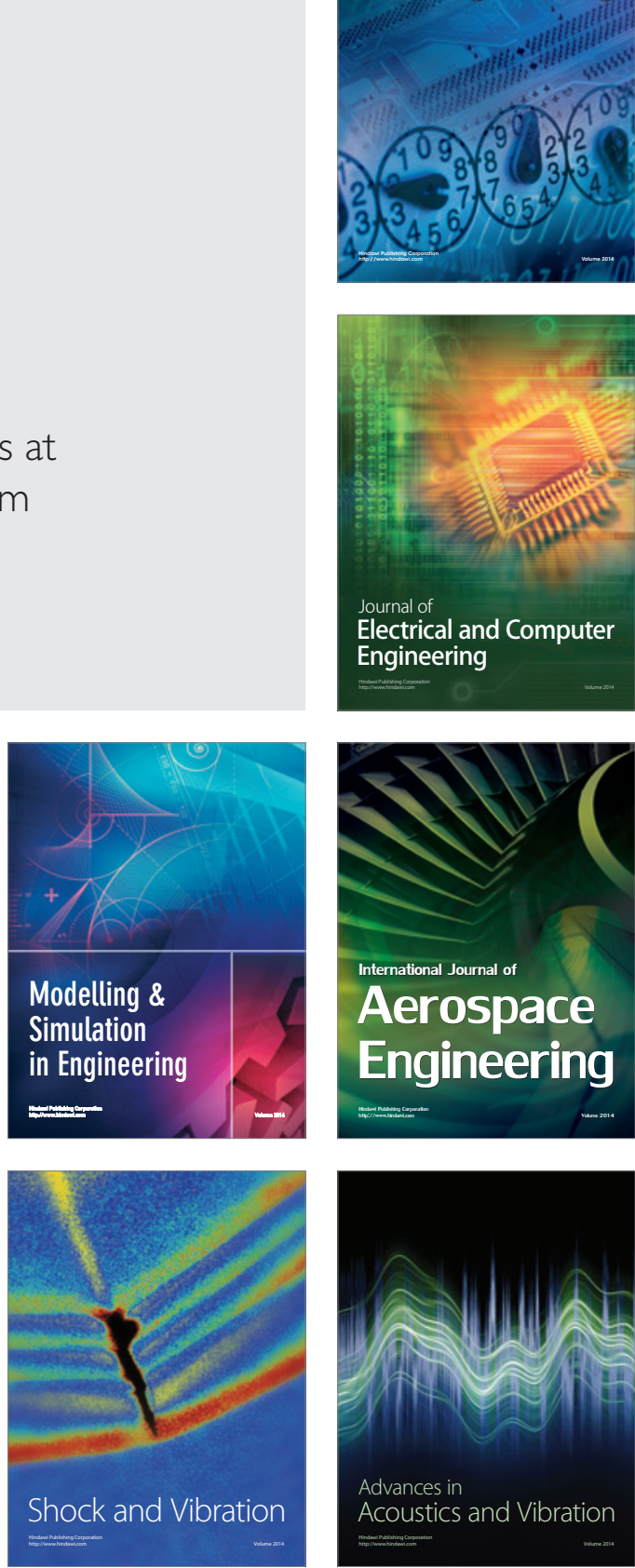